\title{
Clinical effectiveness of virtual reality versus conventional clinic-based vestibular physical therapy on balance and function in active duty service members. A pilot randomized controlled trial
}

\author{
Pinata H. Sessoms ${ }^{1}$. John J. Fraser ${ }^{1,2}$. Dawn M. Bodell ${ }^{1,2,3} \cdot$ Kathrine S. Haluch $^{1,5}$. Amanda E. Markham ${ }^{1,3}$. \\ Jacob S. Brookfield ${ }^{1,3} \cdot$ Jason Jameson ${ }^{1,3} \cdot$ Kim R. Gottshall $^{3,4}$
}

Received: 28 June 2020 / Accepted: 5 June 2021 / Published online: 30 July 2021

This is a U.S. government work and not under copyright protection in the U.S.; foreign copyright protection may apply 2021

\begin{abstract}
Virtual reality (VR) may be useful during rehabilitation of service members with persistent vestibular impairment following concussion. Thirty-eight active duty US military service members with persistent balance impairment resulting from concussion were randomized into three groups [Conventional Vestibular Physical Therapy (CVPT, $n=13$ ), Virtual Reality Vestibular Physical Therapy (VRVPT, $n=12$ ), and Hybrid Virtual Reality and Conventional Vestibular Physical Therapy (HybridVPT, $n=13$ )] and were treated twice weekly for 6 weeks. Changes in clinical measures such as Activities-specific Balance Confidence (ABC) Scale, Dizziness Handicap Inventory (DHI), Functional Gait Assessment (FGA), and Sensory Organization Test (SOT) were assessed from pre-, mid-, and post-treatment scores. A significant main time effect was observed demonstrating clinical improvement over time (ABC: $p<.001, \eta_{p}^{2}=.54$; DHI: $p<.001, \eta_{p}^{2}=.57$; FGA: $p<.001$, $\eta_{p}^{2}=.74$; SOT: $p<.001, \eta_{p}^{2}=.35$ ). Both CVPT and HybridVPT groups demonstrated significant improvements in patientreported confidence and function earlier in the treatment course $(p<.005)$. FGA significantly and incrementally improved at each assessment time point in all treatment groups. The SOT significantly improved early in treatment in the CVPT group only and pre-to-post-treatment in the CVPT and VRVPT groups only. The HybridVPT group did not demonstrate any significant improvement with time in the instrumented SOT measure. In the comparison of pre-to-post-effects of VRVPT and HybridVPT effects compared to CVPT, there was no clear superiority or inferiority observed in either of the experimental treatments. This preliminary work shows initial efficacy of using VR-based therapy for concussed individuals allowing future work to personalize treatment that may improve adherence and engagement to therapy.
\end{abstract}

Keywords Therapeutics $\cdot$ Data display $\cdot$ Craniocerebral trauma $\cdot$ Physical therapy modalities $\cdot$ Sensation disorders

\section{Background}

Pinata H. Sessoms

Pinata.H.Sessoms.civ@mail.mil

1 Warfighter Performance Department, Naval Health Research Center, 140 Sylvester Road, San Diego, CA 92106, USA

2 Physical Therapy Department, Navy Medicine Readiness and Training Command San Diego, 34800 Bob Wilson Drive, San Diego, CA 92134, USA

3 Leidos, Inc, 4161 Campus Point Court, San Diego, CA 92121, USA

4 Florida Ear and Balance Center, 410 Celebration Place, Kissimmee, FL 34747, USA

5 Eagle Applied Sciences, 1826 N Loop 1604 E \#350, San Antonio, TX 78248, USA
Head injuries incurred from direct or indirect traumatic forces may result in neurological impairments that limit function. Warfighters are a special population at risk for such traumatic events that often occur as blunt or blast forces sustained during military operations. According to the Defense and Veterans Brain Injury Center (DVBIC), over 413,000 service members incurred a head injury between the years 2000 and 2019, with approximately $83 \%$ of all head injuries classified as being mild (DVBIC 2019). Following these injuries, there are varying degrees of physical, cognitive, and emotional impairment that may impede function (Scherer and Schubert 2009). Visual and vestibular impairments such 
as blurry vision, postural imbalance, vertigo, headache, and dizziness constitute the most common sequela seen in this population (Johnson et al. 2014; Hoffer et al. 2004). These impairments contribute to degradation of function by limiting activity and restricting participation in community and duty-related tasks. Most individuals who sustain a concussion are expected to recover within 2-3 weeks (Anderson et al. 2006). While this may be reassuring, anywhere from 5 to $58 \%$ of individuals who sustain a concussion will have persistent impairments, to include dizziness and balance deficit (Quatman-Yates et al. 2020, Howell et al. 2018).

Current clinical practice guidelines recommend the evaluation and treatment of head injury earlier in the injury course, to include exercises used to mitigate dizziness and improve postural control, in order to maximize function (Quatman-Yates et al. 2020). Individualized exercise programs that include gaze stabilization, postural stability, gait training, and desensitization of head or body motion during function are often indicated (Alsalaheen et al. 2010; Gottshall and Sessoms 2015). Gaze stabilization exercises are used to correct visual disturbances by training the oculomotor coordination and the vestibulo-ocular reflex to stabilize gaze while the head or body is in motion. Somatosensory exercises improve postural control by challenging the vestibulo-spinal reflexes that control muscle responses. Desensitization exercises mitigate dizziness and motion sensitivity through graded exposure that with time and repetition habituate the individual to the evoking stimuli. When performed as a comprehensive rehabilitation program, these vestibular exercises can help decrease dizziness and risk of falls by improving postural stability, sensory organization, and integration. (Cawthorne 1944; Horak et al. 1992; Shepard et al. 1993). The clinical benefit of vestibular rehabilitation techniques for the treatment of vestibular impairment is well established (Basford et al. 2003; Gottshall 2011; Badke et al. 2005).

As new technologies emerge, healthcare practitioners are provided with new and innovative approaches for assessment, treatment, and the ability to more effectively track clinical outcomes with greater precision. Immersive virtual reality (VR) technologies allow participants to practice challenging balance tasks simultaneously with cognitive tasks in an interactive environment, similar to what might be encountered in real-world settings. Providing patients with head injury with more ecologically valid rehabilitation tasks in a simulated environment may facilitate improved function and confidence when resuming activities of daily living in the community, as well as duty requirements following return to work. New evidence suggests interventions that employ VR are effective for improving balance and confidence and are recommended in the treatment of individuals with vestibular dysfunction (Alahmari et al. 2014; Bergeron et al. 2015; Whitney et al. 2015). The combination of conventional clinic-based therapy augmented with treatment provided using an immersive VR environment (iVRE) may have substantive clinical benefit for the treatment of vestibular impairment following concussion (Gottshall and Sessoms 2015). While VR may be an augment for treatment of service members with vestibular and balance impairment following concussion, little is known about the clinical effectiveness of using VR modalities in lieu of conventional therapeutic methods. Given the potential benefits of these new techniques, it is important to determine whether incorporating VR (either as a supplement or replacement) leads to rates of improvement that are comparable to conventional therapy, or at least does not undermine patient recovery. Therefore, the purpose of this study is to evaluate the clinical effectiveness of iVRE compared to conventional clinic-based vestibular physical therapy on balance and function in a sample of military health care beneficiaries with vestibular impairment following concussion.

\section{Methods}

\subsection{Design}

A pilot parallel randomized controlled trial was performed assessing the independent variables of treatment [Conventional Vestibular Physical Therapy (CVPT), Virtual Reality Vestibular Physical Therapy (VRVPT), Hybrid Virtual Reality and Conventional Vestibular Physical Therapy (HybridVPT)] and time [baseline assessment (T1), interim assessment following 6 treatment visits (T2), final assessment following 12 treatment visits (T3)] on the outcomes of instrumented, clinical, and patient-reported measures of balance and function in military health care beneficiaries with persistent vestibular deficit following concussion.

\subsection{Participants}

US military service members and dependents between the ages of 18 and 55 years with a diagnosis of persistent central vestibular impairment within twelve months following concussion were screened and recruited from a military medical center vestibular clinic from September 2011 to August 2015. Participants were included if they demonstrated substantial vestibular deficit and activity limitation on the Sensory Organization Test (composite score $<70$ ), the Activities-specific Balance Confidence Scale (score $<67 \%$ ), the Dizziness Handicap Inventory (score $>30$ ), and/or the Functional Gait Assessment (score <22). Participant demographics are detailed in Table 1. Individuals with severe physical impairments (inability to stand or walk community distances), who were pregnant, or who were unable to provide informed consent were excluded from the study. Since 
Table 1 Participant demographics

\begin{tabular}{lllllll}
\hline Group & Age (years) & Height $(\mathrm{cm})$ & Weight $(\mathrm{kg})$ & $\begin{array}{l}\text { Time since } \\
\text { injury (days) }\end{array}$ & $\begin{array}{l}\text { Participants report- } \\
\text { ing prior injury }(n)\end{array}$ & $\begin{array}{l}\text { Prior injuries } \\
\text { incurred* }(n)\end{array}$ \\
\hline Mean $(\mathrm{SD})$ & & & & & 2.60 \\
$\mathrm{CVPT}$ & 27.15 & 181.90 & 85.00 & 133.75 & 5 & $(1.52)$ \\
$\mathrm{n}=13$ & $(5.79)$ & $(10.45)$ & $(13.03)$ & $(85.03)$ & & 4.25 \\
$\mathrm{VRVPT}$ & 28.58 & 178.86 & 81.97 & 172.50 & 4 & $(3.95)$ \\
$\mathrm{n}=12$ & $(5.73)$ & $(7.92)$ & $(13.36)$ & $(82.99)$ & & 4.67 \\
HybridVPT & 32.23 & 172.52 & 86.40 & 198.54 & 6 & $(7.61)$ \\
$\mathrm{n}=13$ & $(5.43)$ & $(9.07)$ & $(12.50)$ & $(84.78)$ & & \\
\hline
\end{tabular}

$C V P T$ conventional vestibular physical therapy, VRVPT virtual reality-based vestibular physical therapy, HybridVPT hybrid virtual reality and conventional vestibular physical therapy

*Only includes participants that reported prior head injury to the one for which they were seeking treatment

this was a pilot trial, sample size estimates were not calculated a priori and a sample of convenience was employed. Additionally, the observed sample size per group follows recommended guidelines for pilot studies (Julious 2005) and is similar to sample sizes used in similar previous research (Basford et al 2003; Badke et al 2005). All participants that met inclusion criteria provided informed consent. This study was approved by the Naval Health Research Center's Institutional Review Board in accordance with the ethical standards of the institutional and national research committee and with the 1964 Helsinki Declaration and its later amendments or comparable ethical standards. Study procedures and outcomes were unchanged following the initiation of the study. A CONSORT diagram detailing participant recruitment, allocation, follow-up, and analysis is detailed in Fig. 1.

\subsection{Interventions}

Clinical interventions for all participants, regardless of treatment group, consist of a total of 12 therapy sessions performed twice weekly over a 6-week period. Each session includes approximately 30 min of therapy with scheduled rests between tasks, with treatment sessions lasting approximately $60 \mathrm{~min}$ total. Evidence establishes that improvements in symptoms and physical limitations generally occur with 4-12+ weeks of therapy (Quatman Yates et al. 2020, Gottshall et al. 2010, 2011). Simple randomization was used to equally allocate participants into one of three intervention arms: Conventional Vestibular Physical Therapy (CVPT), which serves as the standard of care control, a Virtual Reality-based Vestibular Physical Therapy (VRVPT), and a Hybrid Virtual Reality and Conventional Vestibular Physical Therapy (HybridVPT) that employs both intervention approaches. While participants in CVPT and VRVPT receive exclusively the allocated intervention, the HybridVPT group receives one day of CVPT and one day VRVPT per week for the study duration. Blinding of the clinicians and participants and allocation concealment was not feasible in this pilot trial.

The CVPT group is provided standardized treatment at the military medical center vestibular therapy clinic, a comprehensive balance and spatial disorientation clinic that specializes in the evaluation and treatment of injured service members (Gottshall et al. 2005; Hoffer et al. 2003). The rehabilitation course utilized in this study is based on clinical best practices now reflected in practice guidelines (Quatman-Yates et al. 2020) and consists of several vestibular ocular, gaze stabilization, and physical balance tasks prescribed by the evaluating physical therapist to target specific deficits identified on physical examination, while considering patient preference and priorities. The treatment protocol includes one or many exercises that leverage eyes open and closed conditions, single and double leg stances, stable and unstable surfaces, and internal and external perturbations to challenge the participant (Table 2).

The VRVPT leverages the use of the Computer Assisted Rehabilitation Environment (CAREN Extended, Motekforce Link BV, Amsterdam, Netherlands), a large-scale iVRE located in a research center in proximity to the medical center. The CAREN system employs hardware and software capabilities that challenge participants both physically and cognitively, with the capability to collect objective data in a safe environment. It is comprised of an instrumented treadmill embedded on a platform that can move in six degrees of freedom. The platform is surrounded by a panoramic screen that immerses participants in a virtual reality (VR) scenario. These capabilities, working synchronously, allow participants to engage in realistic tasks applicable to their specific needs. The VR vestibular therapy in this experiment includes clinical aspects of rehabilitation (balance, vestibulo-ocular reflex, gaze stability) through physical and cognitive challenges. Standard conventional clinical therapy methods for individuals with vestibular dysfunction are employed as the foundation of the VR applications. While the VR environment allows for innovative delivery of those therapies, 
Table 2 CVPT exercises

\begin{tabular}{lll}
\hline Static stance exercises & Dynamic exercises & Gaze stabilization and oculomotor \\
\hline $\begin{array}{l}\text { Double or single limb static standing on a stable } \\
\text { surface with eyes open and closed }\end{array}$ & Double or single limb stance rebound ball throwing & Vestibulo-ocular reflex $\times 1$ viewing \\
$\begin{array}{c}\text { Double or single limb static standing on a compliant } \\
\text { surface with eyes open and closed }\end{array}$ & Agility & Vestibulo-ocular reflex $\times 2$ viewing \\
$\begin{array}{c}\text { Anterior/posterior and medial/lateral weight shifting } \\
\text { on a rocker-board with eyes open and closed }\end{array}$ & Lunges, squats, kettle bell exercises & Smooth pursuit \\
$\begin{array}{c}\text { Romberg/Sharpened Romberg on compliant surface } \\
\text { with eyes open and closed }\end{array}$ & Proprioceptive neuromuscular facilitation & Saccade \\
& Walking program for cardiovascular endurance & \\
\hline
\end{tabular}

the clinical basis of the VR therapy parallels conventional therapy.

The tasks employed for VRVPT include iVRE scenarios that challenge the participant during static stance by shifting the center of mass to the limits of stability and by facilitating weight shifting. During dynamic tasks such as walking, the pitch and roll of the motion platform during walking changes to follow the virtual terrain. The level of difficulty for each application is tracked and incrementally increased as participants improve with the task. All applications challenge multisensory modalities, cognition, and motor function with the use of dual and multitasking during treatment. Cognitive tasks include decision-making, scanning, and target identification. Prior to engaging in therapy, subjects are given a brief period of familiarization (i.e., treadmill walking within the iVRE) to ensure their comfort prior to starting each session. The iVRE applications used in treatment of the VRVPT and HybridVPT groups are:

1. Continuous Road-introduction to ambulation in the environment. Starts with level walking at a self-selected pace. Velocity, duration of activity, and platform motion can be adjusted. Cognitive tasks are included for dual tasking as well as for encouraging head turning. Motion platform perturbations are introduced in multiple directions for challenging proprioceptive balance.

2. Forest Road-uses upper extremities during ambulation. Moving targets appear at random intervals while the subject negotiates undulating terrain. The motion platform moves synchronously with the terrain. This scenario promotes internal perturbation through reaching, while platform movement provides external perturbation.

3. Combat mountain walk-distractions enhance the complexity of ambulation in a virtual environment by providing stimulation through a multisensory experience. Interactive objects that the subject must look for and target, as well as passing vehicles and aircraft enhance auditory and visual stimuli. The motion platform moves synchronously with the terrain. Targeting challenges keeping the head stable while the body is moving.

4. Boat-challenges timing, sequencing, anticipatory movements, and endurance while the participant maintains balance on a dynamic platform. Participants shift their weight on the platform to control a boat on the screen and navigate it through a course of buoys. The motion platform moves synchronously with the boat over the waves.

Further details pertaining to the exercises used in both the CVPT and VRVPT groups, including details of the VR scenarios, are described in greater detail by Haluch et al. (2020). The specific applications use targeted aspects of vestibular dysfunction in similar yet novel ways to those of standard clinical therapy. Though the VR applications' visual environment are different from those of CVPT, the VR methods address vestibular deficits in parallel ways to conventional methods.

\subsection{Outcome measures}

Completion of patient-reported outcome measures, instrumented measures, and clinical assessments were performed prior to treatment initiation (T1), at mid-treatment following six treatment sessions (T2), and post-treatment following 12 sessions (T3). All outcomes were assessed at the military medical center vestibular therapy clinic.

\subsubsection{Patient-reported outcomes}

Patient-reported outcome measures included the Activitiesspecific Balance Confidence Scale and the Dizziness Handicap Inventory. The Activities-specific Balance Confidence Scale $(\mathrm{ABC})$ is a patient-reported outcome measure developed to gauge the psychological impact of balance impairment and/or falls. It is a self-report of balance confidence in daily activities. Participants score 16 items on a scale of $0 \%$ (no confidence) to $100 \%$ (total confidence) that they can 
perform the activity without losing balance. A score of less than $67 \%$ is considered to be indicative of greater fall risk for those with vestibular disorders (Lajoie and Gallagher 2004). The ABC has been found to be internally consistent, reliable, and valid (Powell and Myers 1995).

The Dizziness Handicap Inventory (DHI) consists of 25 items and was developed to evaluate activity limitation and participation restriction resulting from dizziness. Answers for each item are graded 0-4 with total score ranges from 0 to 100 , with 100 signifying the highest degree of impairment. This patient-reported outcome measure grades levels of perceived handicap; a score of 0-30 indicates mild impairment, 31-60 indicates moderate impairment, and 61-100 indicates severe impairment (Whitney et al 2004). The DHI has been validated previously (Newman and Jacobson 1993) and is sensitive to subtle balance deficits following mild head injury (Gottshall et al. 2003).

\subsubsection{Instrumented and clinical outcome measures}

Physical clinical assessments included the Sensory Organization Test and the Functional Gait Assessment. The Sensory Organization Test (SOT) is an instrumented six-condition test performed using Computerized Dynamic Posturography (Neurocom International, Inc., Clakamas, OR). The SOT identifies abnormalities in the three sensory systems that contribute to postural control: somatosensory, visual, and vestibular. The system analyzes the individual's ability to maintain upright standing balance in six different conditions. A composite score is generated from six trials, two trials of each balance condition. Scores near 100 represent little sway and scores near 0 represent excessive sway. Computerized Dynamic Posturography has been shown to be reliable by Basford et al. (2003). For this study, if participants rapidly progressed in the first half of the treatment course and exceeded a clinically meaningful threshold of $70 \%$ on the SOT composite during $\mathrm{T} 2$, that score was carried over to the final measurement. This was performed in accordance with standard clinic procedures and under the assumption that in the absence of further neurological insult, participants would not have functional decline in sensory organization between time points.

The Functional Gait Assessment (FGA) is a set of ten gait tasks with a standardized grading system that is administered and scored by a physical therapist trained in evaluation of this test. Scoring is based on ability to walk over a level surface, change speed, walk while turning the head, walk and pivot, step over an obstacle, walk with a narrow base of support (heel to toe), walk with eyes closed, walk backward, and walk up steps. A score of 3 is possible for each task for a total composite score of 30 . A score of 30 indicates no difficulty with gait, while a score of 0 represents severe difficulty with gait. A score of 22 or less has indicated an increased risk for falls in community dwelling older adults (Wrisley et al. 2004).

\subsection{Statistical analysis}

Descriptive statistics were calculated for demographic information. To assess the effects of treatment and time on the outcome measures, linear mixed-effects ANOVAs were run to account for unbalanced data across conditions as well as missing data points on the SOT test $(n=8)$. Analyses were conducted and $p$-values were calculated using R (R Core Team 2012) and the anova.lme(lme()) function within the package nmle (Pinheiro et al. 2019). Fixed effects of Group, Time, and the Group x Time interaction were entered for each outcome measure, in addition to a random effect of Subject. In order to examine post hoc mean score differences, paired sample t-tests (for comparisons between time points) and two sample t-tests (for comparisons between groups) were performed. These $\mathrm{t}$-tests were completed using the package rstatix (Kassambara 2019). In order to adjust $p$-values for the increased false discovery rate that comes with making multiple comparisons, a Benjamini-Hochberg (BH) correction was applied to each set of post hoc tests.

To explore potential differences in relative effectiveness, the pre-to-post-change scores for VRVPT and HybridVPT were separately compared to the pre-to-post-change scores for CVPT. To substantiate the robustness of any conclusions drawn from the relatively small sample size employed in this pilot study, multiple approaches to constructing confidence intervals were employed to include bootstrapping methods (for an accessible overview, see Chong and Choo 2011). Bootstrapping methods are computationally intensive methods that involve resampling with replacement, many times, from a data set and are effective at estimating the standard deviation of a given statistic's sampling distribution (i.e., the standard error). Using such an approach requires fewer assumptions than other estimation approaches, and one generally does not need to know the mathematical form of the sampling distribution.

All such analyses were carried out in R, using the packages effsize (Torchiano 2017) and bootES (Gerlanc and Kirby 2015), with the number of bootstrap replicates set to 2,000 . All reported bootstrapped $95 \%$ confidence intervals were corrected for bias and skewness in the distribution of bootstrapped estimates (bias corrected and acceleratedBCa-see Efron 1987). In particular, the Hedges's $g$ effect size and $95 \%$ confidence intervals were calculated using both actual sample size and bootstrapping methods. Hedges's $g$ is a small sample correction of Cohen's $d$, a widely used standardized mean difference, and measure of effect size. 


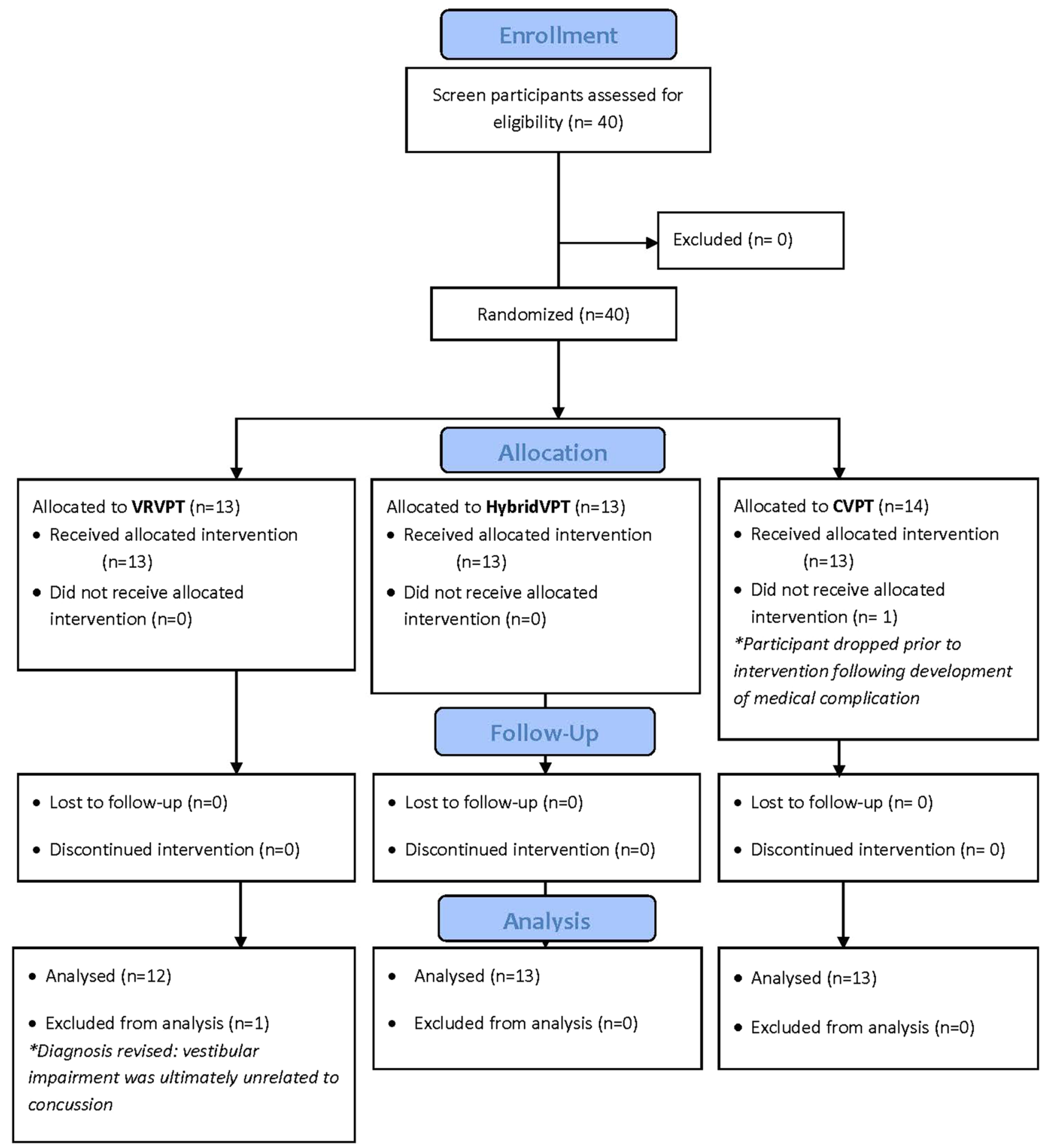

Fig. 1 CONSORT diagram detailing participant recruitment, allocation, follow-up, and analysis

\section{Results}

Descriptive statistics for age, height, weight, days since injury, and number of previous head injuries are presented by group and reported in Table 2. Between one-third and one-half of the participants reported at least one prior head injury to the one for which they were seeking treatment ( 5 in CVPT, 4 in VRVPT, and 6 in HybridVPT). For those reporting a prior head injury, the average number of prior head injuries were $2.60(\mathrm{SD}=1.52)$ for CVPT, $4.25(\mathrm{SD}=3.95)$ for VRVPT, and $4.67(\mathrm{SD}=7.61)$ for HybridVPT groups. There were no significant differences 
between groups on time since injury, number of previous injuries, or other demographic variables except for height ( $p=.04$ ), with the HybridVPT group found to be significantly shorter than the CVPT group [mean difference $\left.\left(M_{\text {diff }}\right): 9.5 \mathrm{~cm}, p=.02\right]$. No other differences were found between groups for any demographic variables.

Table 3 and Figs. 2, 3, 4 and 5 detail the effects of time and treatment for the four outcomes of interest. While there were no significant Group main effects or Group $\mathrm{x}$ Time interactions, post hoc comparisons were conducted to evaluate when participants improved (Table 4) and the magnitude of difference in change scores (Fig. 6). A significant main time effect was observed demonstrating clinical improvement over time (SOT: $p<.001, \eta^{2}{ }_{p}=0.35$; ABC: $p<.001, \eta^{2}{ }_{p}=0.54$; FGA: $p<.001, \eta_{p}^{2}=0.74$; DHI: $\left.p<.001, \eta^{2}=0.57\right)$. Both CVPT and HybridVPT groups demonstrated significant improvements in patientreported confidence and function earlier in the treatment course $(p<.005)$, in contrast to the VRVPT group that did not. Clinician-assessed FGA significantly and incrementally improved at each assessment time point in all treatment groups. The instrumented SOT scores significantly improved between pre-to-mid-time points in the CVPT group only and pre-to-post-time points in the CVPT and VRVPT groups. The HybridVPT group did not demonstrate any significant improvement with time in the instrumented SOT measure. In the comparison of pre-to-post-effects, VRVPT and HybridVPT effects compared to CVPT, there was no clear superiority or inferiority observed in either of the experimental treatments observed (Fig. 6).

\section{Discussion}

The primary findings of this pilot study suggest that CVPT, VRVPT, or a combination of both interventions result in improvement in patient-reported, clinician-assessed, and instrumented measures of self-reported dizziness handicap and balance confidence, functional balance, and functional gait following six weeks of treatment. This study also suggests that rehabilitation within an iVRE is able to achieve similar outcomes to conventional vestibular physical therapy for participants with persistent vestibular impairments following concussion.

Vestibular therapy mitigates dizziness and improves impairments in postural control resulting from concussive injuries (Alsalaheen et al. 2010). VR is an evolving technology that has been used as a viable option for treating such balance dysfunction, and is being used to treat impairments across multiple physical therapy domains (Levin et al. 2015; Cuthbert et al. 2014; Howard 2017; Whitney et al. 2006). There are some potential benefits for the use of an iVRE as a treatment modality for therapy, such as the ability to easily administer engaging dual tasks during therapy sessions. Dual task training has been shown to benefit those who suffer from sensory impairments after sustaining concussion (Hopin et al. 2013, Teel et al. 2013, Lee et al. 2013). Simultaneously administering physical and cognitive workloads
Table 3 Effects of treatment and time on balance and function

\begin{tabular}{|c|c|c|c|c|c|c|}
\hline & \multicolumn{3}{|l|}{ Mean (SD) } & \multirow[t]{2}{*}{ Effect } & \multirow[t]{2}{*}{$p$} & \multirow[t]{2}{*}{$\eta_{p}^{2}$} \\
\hline & Baseline T1 & $\mathrm{T} 2$ & $\mathrm{~T} 3$ & & & \\
\hline \multicolumn{7}{|c|}{ Sensory Organization Test (SOT) } \\
\hline CVPT & $60.17(16.19)$ & $72.69(13.03)$ & $79.08(10.31)$ & Group & 0.59 & 0.03 \\
\hline VRVPT & $64.08(14.37)$ & $68.75(15.9)$ & $74.25(11.74)$ & Time & $<0.001$ & 0.35 \\
\hline HybridVPT & $69.69(13.70)$ & $76.08(10.19)$ & $76.77(14.06)$ & Group*Time & 0.15 & 0.09 \\
\hline \multicolumn{7}{|c|}{ Activity-specific Balance Confidence (ABC) } \\
\hline CVPT & $72.00(16.55)$ & $81.38(11.13)$ & $90.62(7.46)$ & Group & 0.11 & 0.12 \\
\hline VRVPT & $75.50(11.84)$ & $80.34(11.74)$ & $90.05(5.58)$ & Time & $<0.001$ & 0.54 \\
\hline HybridVPT & $66.38(16.20)$ & $73.08(15.32)$ & $82.00(10.57)$ & Group*Time & 0.86 & 0.02 \\
\hline \multicolumn{7}{|c|}{ Functional gait assessment (FGA) } \\
\hline CVPT & $24.46(2.11)$ & $27.46(1.76)$ & $29.62(0.96)$ & Group & 0.80 & 0.01 \\
\hline VRVPT & $25.25(3.28)$ & $28.33(2.50)$ & $29.33(1.72)$ & Time & $<0.001$ & 0.74 \\
\hline HybridVPT & $24.77(3.09)$ & $28.46(2.11)$ & $29.38(1.19)$ & Group*Time & 0.47 & 0.05 \\
\hline \multicolumn{7}{|c|}{ Dizziness Handicap Inventory (DHI) } \\
\hline CVPT & $41.69(21.29)$ & $31.00(17.13)$ & $20.69(16.48)$ & Group & 0.72 & 0.02 \\
\hline VRVPT & $36.42(14.93)$ & $35.42(21.83)$ & $18.92(18.01)$ & Time & $<0.001$ & 0.57 \\
\hline HybridVPT & $46.69(16.17)$ & $37.23(14.64)$ & $20.54(9.79)$ & Group*Time & 0.30 & 0.07 \\
\hline
\end{tabular}

$C V P T$ conventional vestibular physical therapy, VRVPT virtual reality vestibular physical therapy, Hybrid$V P T$ hybrid virtual reality and conventional vestibular physical therapy 

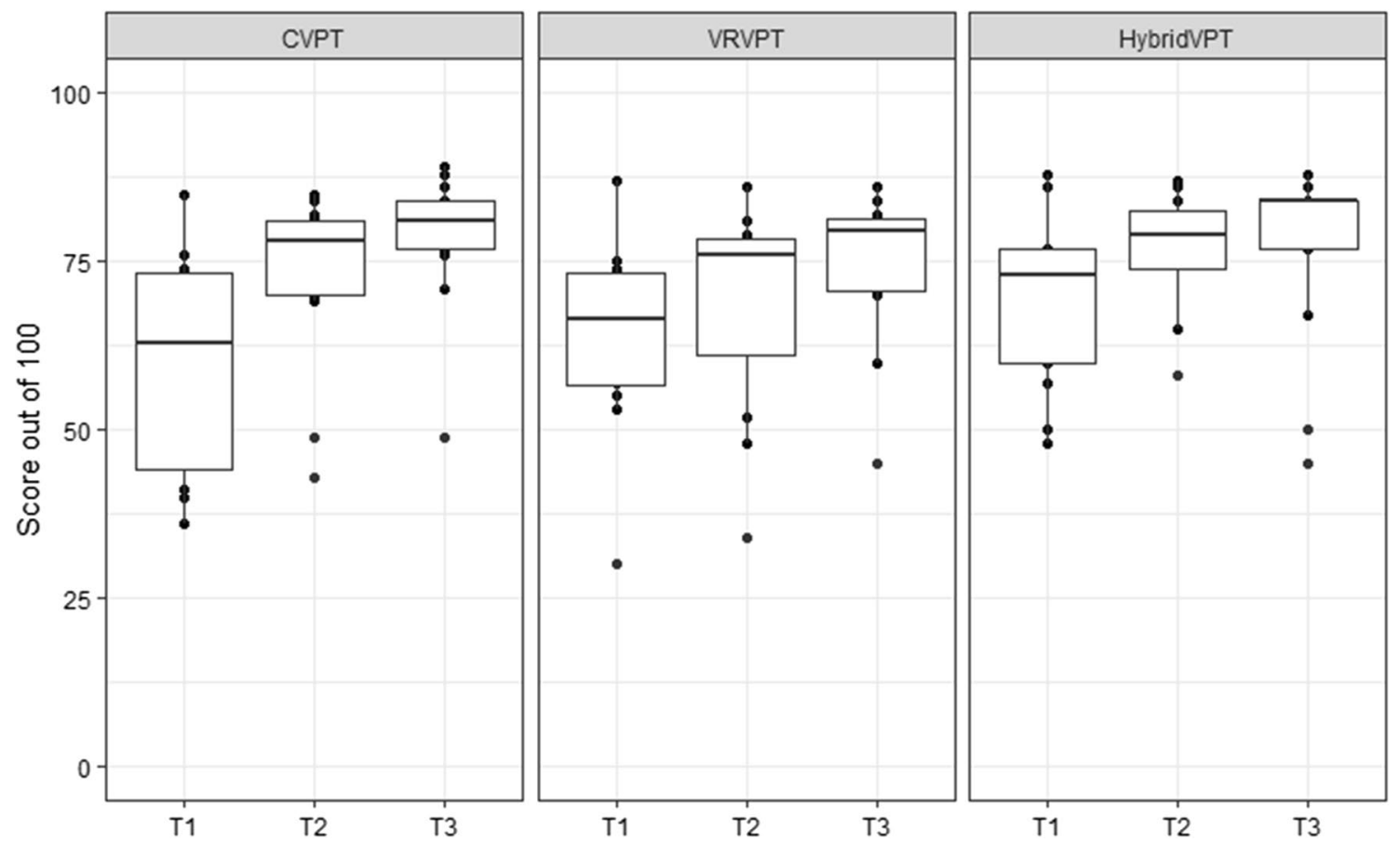

Fig. 2 Sensory Organization Test (SOT) Scores by condition. Dots depict individual subject scores. Boxes depict the interquartile range (IQR; 25th-75th percentile) of scores with the thick horizontal line depicting the median score for that group. Whisker lines outside of

the boxes extend to the score at or nearest outside 1.5 times the IQR. $C V P T$ Conventional vestibular physical therapy, VRVPT virtual reality vestibular physical therapy, HybridVPT hybrid virtual reality and conventional vestibular physical therapy
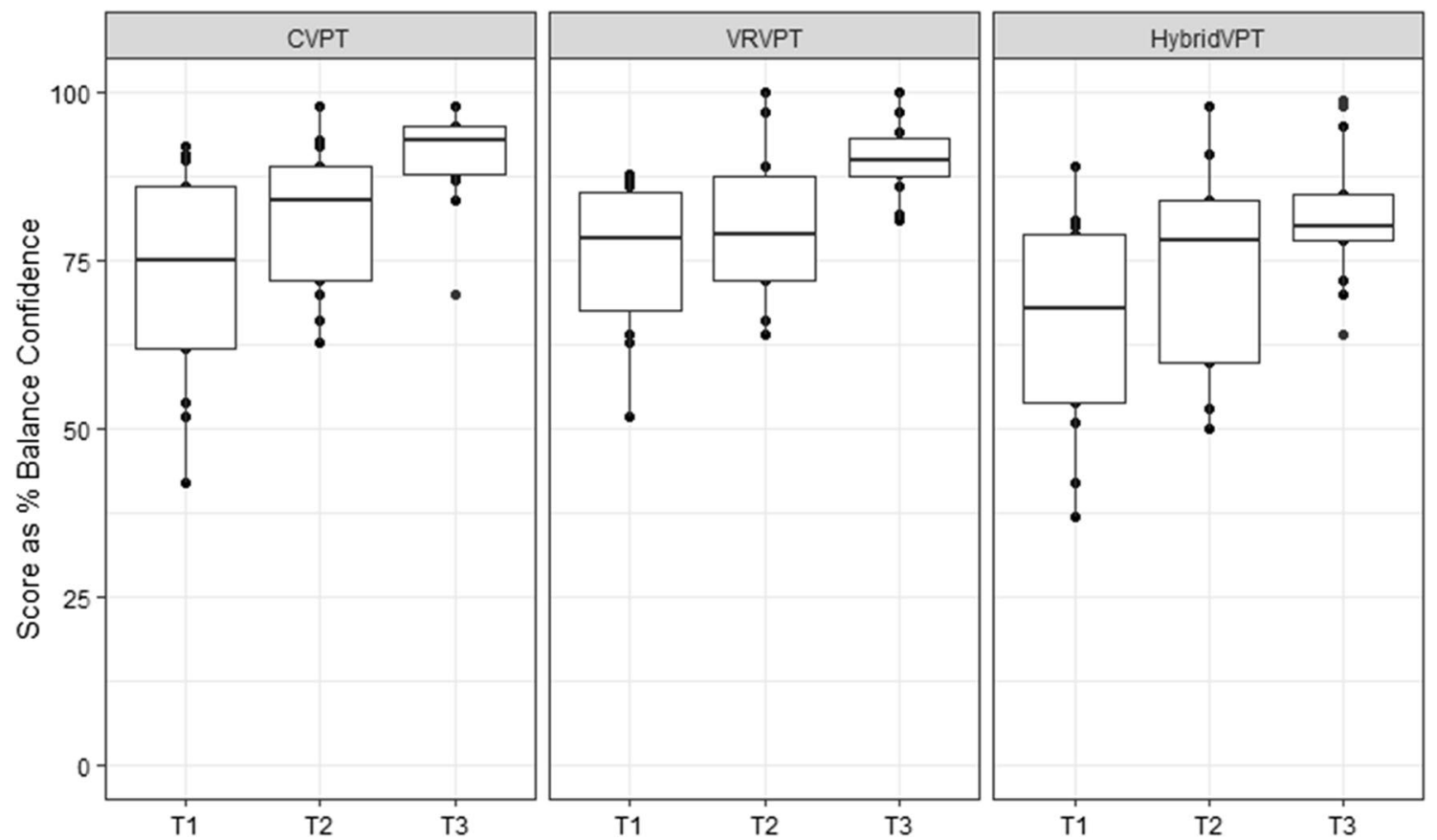

Fig. 3 Activity-specific Balance Confidence (ABC) scores by condition. Dots depict individual subject scores. Boxes depict the interquartile range (IQR; 25th-75th percentile) of scores with the thick horizontal line depicting the median score for that group. Whisker lines outside of the boxes extend to the score at or nearest outside 1.5 times the IQR. CVPT conventional vestibular physical therapy, VRVPT virtual reality vestibular physical therapy, HybridVPT hybrid virtual reality and conventional vestibular physical therapy 

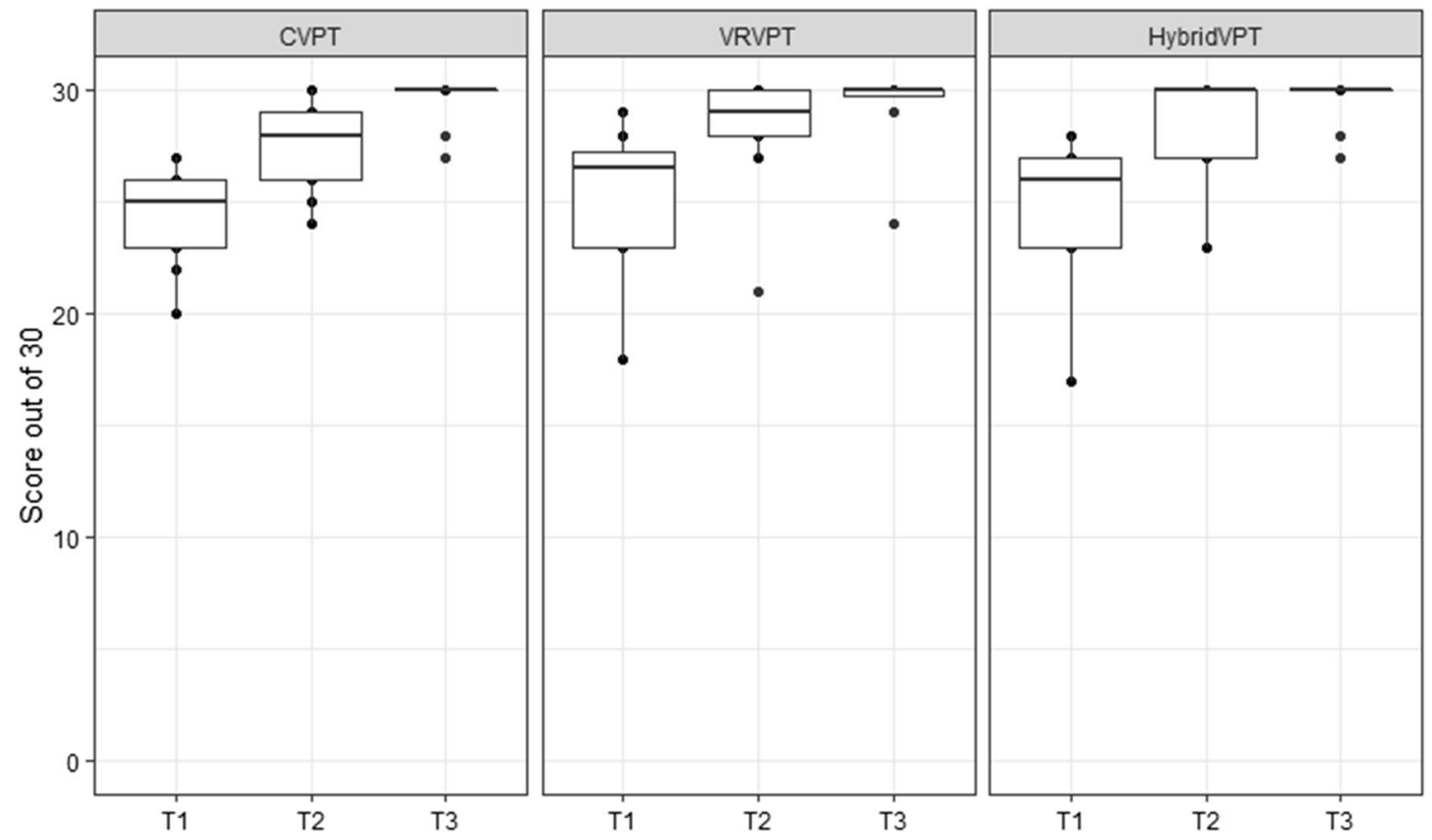

Fig. 4 Functional Gait Assessment (FGA) scores by condition. Dots depict individual subject scores. Boxes depict the interquartile range (IQR; 25th-75th percentile) of scores with the thick horizontal line depicting the median score for that group. Whisker lines outside of the boxes extend to the score at or nearest outside 1.5 times the IQR. $C V P T$ conventional vestibular physical therapy, VRVPT virtual reality vestibular physical therapy, HybridVPT hybrid virtual reality and conventional vestibular physical therapy
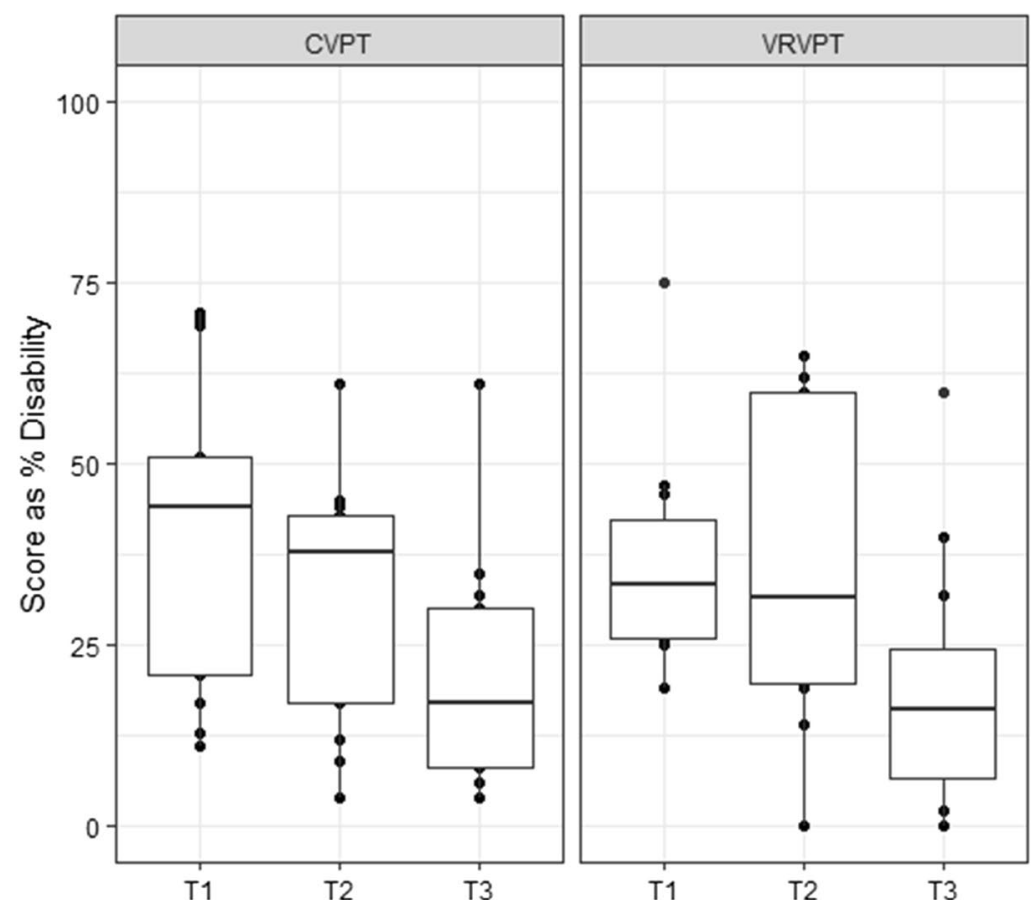

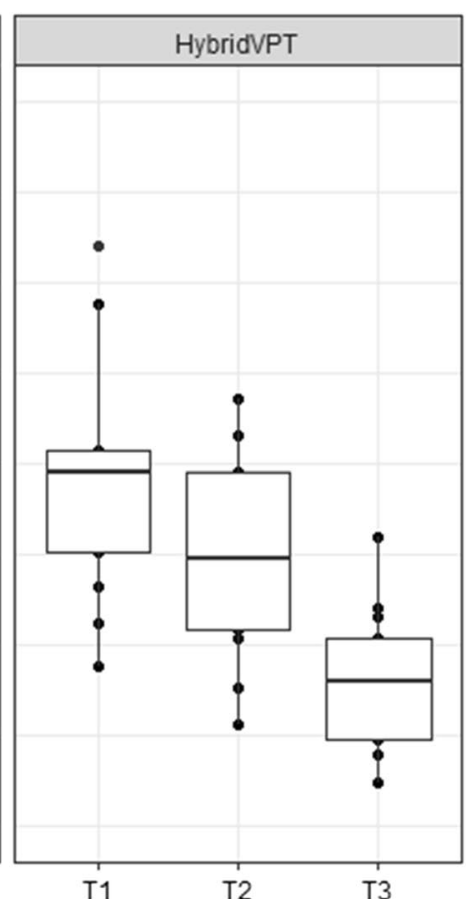

T1
Fig. 5 Dizziness Handicap Inventory (DHI) scores by condition. Dots depict individual subject scores. Boxes depict the interquartile range (IQR; 25th-75th percentile) of scores with the thick horizontal line depicting the median score for that group. Whisker lines outside of the boxes extend to the score at or nearest outside 1.5 times the IQR. CVPT conventional vestibular physical therapy, VRVPT virtual reality vestibular physical therapy, HybridVPT hybrid virtual reality and conventional vestibular physical therapy 
Table 4 Post hoc within-group comparisons over time

\begin{tabular}{|c|c|c|c|}
\hline & $p$ & $M_{\text {diff }}$ & $95 \% \mathrm{CI}$ \\
\hline \multicolumn{4}{|c|}{ Sensory Organization Test (SOT) } \\
\hline \multicolumn{4}{|l|}{ CVPT } \\
\hline $\mathrm{T} 1$ to $\mathrm{T} 2$ & .004 & 12.50 & {$[5.03,19.97]$} \\
\hline $\mathrm{T} 2$ to $\mathrm{T} 3$ & .11 & 6.38 & {$[-1.58,14.35]$} \\
\hline $\mathrm{T} 1$ to $\mathrm{T} 3$ & .003 & 18.83 & {$[7.66,30.00]$} \\
\hline \multicolumn{4}{|l|}{ VRVPT } \\
\hline $\mathrm{T} 1$ to $\mathrm{T} 2$ & .09 & 4.67 & {$[-0.20,9.53]$} \\
\hline $\mathrm{T} 2$ to $\mathrm{T} 3$ & .10 & 5.50 & {$[-1.13,12.13]$} \\
\hline $\mathrm{T} 1$ to $\mathrm{T} 3$ & .003 & 10.17 & {$[4.37,15.96]$} \\
\hline \multicolumn{4}{|c|}{ HybridVPT } \\
\hline $\mathrm{T} 1$ to $\mathrm{T} 2$ & .16 & 5.67 & {$[-2.71,14.04]$} \\
\hline $\mathrm{T} 2$ to $\mathrm{T} 3$ & .11 & 3.33 & {$[-0.85,7.52]$} \\
\hline $\mathrm{T} 1$ to $\mathrm{T} 3$ & .11 & 7.08 & {$[-1.93,16.08]$} \\
\hline \multicolumn{4}{|c|}{ Activity-specific balance confidence (ABC) } \\
\hline \multicolumn{4}{|l|}{ CVPT } \\
\hline $\mathrm{T} 1$ to $\mathrm{T} 2$ & .004 & 9.38 & {$[4.36,14.41]$} \\
\hline $\mathrm{T} 2$ to $\mathrm{T} 3$ & .02 & 9.23 & {$[1.84,16.62]$} \\
\hline $\mathrm{T} 1$ to $\mathrm{T} 3$ & .004 & 18.62 & {$[7.72,29.51]$} \\
\hline \multicolumn{4}{|l|}{ VRVPT } \\
\hline $\mathrm{T} 1$ to $\mathrm{T} 2$ & .24 & 4.84 & {$[-3.65,13.33]$} \\
\hline $\mathrm{T} 2$ to $\mathrm{T} 3$ & .002 & 9.71 & {$[4.85,14.57]$} \\
\hline $\mathrm{T} 1$ to $\mathrm{T} 3$ & .002 & 14.55 & {$[7.56,21.54]$} \\
\hline \multicolumn{4}{|c|}{ HybridVPT } \\
\hline $\mathrm{T} 1$ to $\mathrm{T} 2$ & .005 & 6.70 & {$[2.38,11.00]$} \\
\hline $\mathrm{T} 2$ to $\mathrm{T} 3$ & .002 & 8.92 & {$[4.28,13.46]$} \\
\hline $\mathrm{T} 1$ to $\mathrm{T} 3$ & .001 & 15.62 & {$[9.27,21.96]$} \\
\hline \multicolumn{4}{|c|}{ Functional gait assessment (FGA) } \\
\hline \multicolumn{4}{|l|}{ CVPT } \\
\hline $\mathrm{T} 1$ to $\mathrm{T} 2$ & .001 & 3.00 & {$[1.50,4.50]$} \\
\hline $\mathrm{T} 2$ to $\mathrm{T} 3$ & .001 & 2.15 & {$[1.05,3.25]$} \\
\hline $\mathrm{T} 1$ to $\mathrm{T} 3$ & $<.001$ & 5.15 & {$[3.58,6.73]$} \\
\hline \multicolumn{4}{|l|}{ VRVPT } \\
\hline $\mathrm{T} 1$ to $\mathrm{T} 2$ & .001 & 3.08 & {$[1.72,4,45]$} \\
\hline $\mathrm{T} 2$ to $\mathrm{T} 3$ & .007 & 1.00 & {$[0.33,1.66]$} \\
\hline $\mathrm{T} 1$ to $\mathrm{T} 3$ & $<.001$ & 4.08 & {$[2.51,5.65]$} \\
\hline \multicolumn{4}{|c|}{ HybridVPT } \\
\hline $\mathrm{T} 1$ to $\mathrm{T} 2$ & $<.001$ & 3.69 & {$[2.72,4.66]$} \\
\hline $\mathrm{T} 2$ to $\mathrm{T} 3$ & .03 & 0.92 & {$[0.08,1.76]$} \\
\hline $\mathrm{T} 1$ to $\mathrm{T} 3$ & $<.001$ & 4.62 & {$[3.12,6.11]$} \\
\hline \multicolumn{4}{|c|}{ Dizziness handicap inventory (DHI) } \\
\hline \multicolumn{4}{|l|}{ CVPT } \\
\hline $\mathrm{T} 1$ to $\mathrm{T} 2$ & .002 & -10.69 & {$[-16.20,-5.18]$} \\
\hline $\mathrm{T} 2$ to $\mathrm{T} 3$ & .002 & -10.31 & {$[-15.92,-4.69]$} \\
\hline $\mathrm{T} 1$ to $\mathrm{T} 3$ & $<.001$ & -21.00 & {$[-28.45,-13.55]$} \\
\hline \multicolumn{4}{|l|}{ VRVPT } \\
\hline $\mathrm{T} 1$ to $\mathrm{T} 2$ & .89 & -1.00 & {$[-16.29,14.29]$} \\
\hline $\mathrm{T} 2$ to $\mathrm{T} 3$ & .02 & -16.50 & {$[-28.66,-4.34]$} \\
\hline $\mathrm{T} 1$ to $\mathrm{T} 3$ & .005 & -17.50 & {$[-26.91,-8.09]$} \\
\hline \multicolumn{4}{|c|}{ HybridVPT } \\
\hline $\mathrm{T} 1$ to $\mathrm{T} 2$ & .005 & -9.46 & {$[-15.48,-3.45]$} \\
\hline
\end{tabular}

Table 4 (continued)

\begin{tabular}{llll}
\hline & $p$ & $M_{\text {diff }}$ & $95 \%$ CI \\
\hline T2 to T3 & $<.001$ & -16.69 & {$[-23.44,-9.94]$} \\
T1 to T3 & $<.001$ & -26.15 & {$[-33.69,-18.62]$} \\
\hline
\end{tabular}

$C V P T$ conventional vestibular physical therapy, VRVPT virtual reality vestibular physical therapy, HybridVPT hybrid virtual reality and conventional vestibular physical therapy, $M_{\text {diff }}$ mean difference, $C I$ confidence interval, $T 1$ baseline assessment, $T 2$, interim assessment following 6 treatment visits, T3 final assessment following 12 treatment visits

on the patient has real-life application to activities of daily living and even return to duty. Depending on the severity of the subject's impairments, iVREs allow therapists to tailor the therapy by singular or dual task with the ability to adjust the timing and intensity of the task. Another useful advantage is that it can objectively analyze functional responses such as gait mechanics and postural control, making information readily available to the clinician to adapt training specific to patient needs. iVREs allow the user to practice life-like situations in a safe environment, which could promote confidence in their ability to return to their activities of daily living and may ultimately reduce the amount of time in therapy. This is particularly desirable when considering the potential for return to duty.

In the evaluation of pre-to-post-treatment effects between groups, CVPT tended to outperform the experimental treatments that encompassed iVRE in most outcomes, with earlier improvements and greater overall treatment effects noted. However, since all values compatible with these comparisons included an effect size of 0 , we cannot yet rule out the possibility that all groups are in fact clinically indistinguishable. Finally, a suggestive trend emerges when comparing the relative effectiveness of the VR and Hybrid groups. The magnitude of treatment effects in the HybridVPT outperformed VRVPT in all but one measure, suggesting that some combination of VR therapy combined with traditional methods could provide a better solution than VR alone. Whether this claim stands up to scrutiny should be explored in future research.

Both HybridVPT and CVPT therapy groups demonstrated greater and more rapid perceived improvement in balance confidence and function in both patient-reported measures compared to the VRVPT group. It is possible that exposure to realistic scenarios in the iVRE may change the patient's perception of their function by sensitizing and contextualizing activity limitation, such as walking on uneven terrain or dual tasking. This may be especially true when social participation is avoided or restricted following injury, limiting their exposure. Another plausible explanation is related to the psychological effects experienced when a patient works with a treating physical therapist in a therapeutic alliance 
Fig. 6 Bootstrapped Hedges's $\mathrm{g}$ effect size estimates and $95 \%$ confidence intervals comparing pre-to-post-changes in balance and function following 12 treatment visits of virtual reality vestibular physical therapy (VRVPT) and hybrid virtual reality and conventional vestibular physical therapy (HybridVPT) compared to conventional vestibular physical therapy

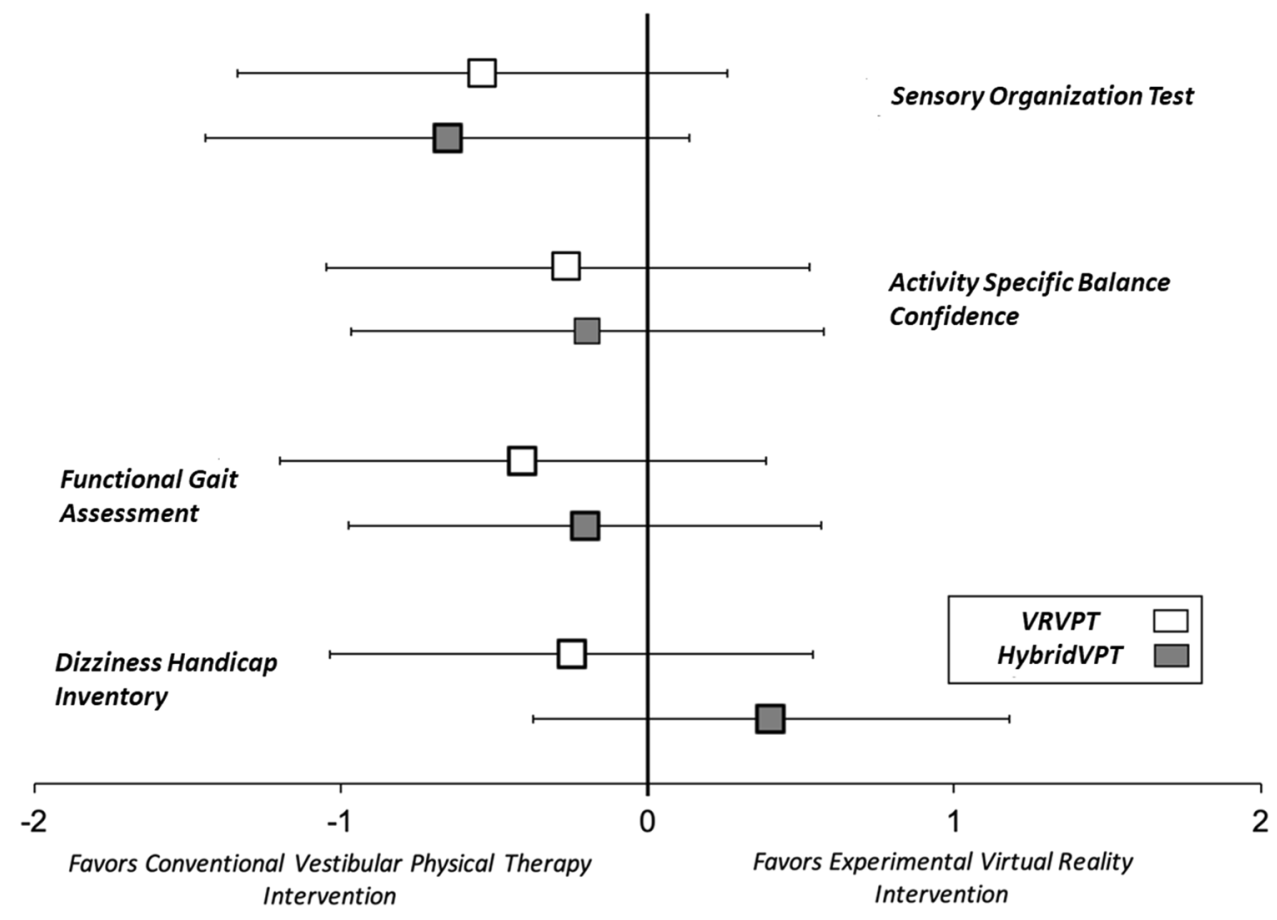

(Hall et al. 2010). There are therapeutic psychological effects, such as improved self-efficacy or confidence, experienced by patients during rehabilitation following injury (Coyassin et al. 2015). It is plausible that participants in the CVPT and HybridVPT groups benefitted from their interactions with the treating physical therapist during care, whereas participants in VRVPT may not have received that same benefit. This supposition warrants future investigation. Specific measures of self-efficacy are not addressed in this pilot study. Though the ABC and DHI assess self-reported confidence and ability of certain activities, standardized measures, such as Albert Bandra's General Self-Efficacy Scale, or other scale-based questionnaires were not included.

Clinician-assessed FGA scores improved for all three groups at each time point. No group differences were seen at any specific time point suggesting that all three therapy methods were similarly successful in improving gait characteristics. Notably, group means approximated the ceiling of a score of 30 by the third assessment (T3). Whether this is a function of ceiling effects of the assessment tool or impairment severity in this study sample is unclear. The Community Balance and Mobility Scale (Pape et al. 2019) was developed in the recent years, which may be a more sensitive tool to detect balance and gait deficits in this population. Lastly, the assessing clinicians in this pilot study were not blinded to group assignment. Since the FGA is reliant on clinician judgment and scoring, detection bias cannot be ruled out despite intentions for absolute objectivity.

While there was a significant main effect in SOT performance with time, only the VRVPT and CVPT groups demonstrated statistically significant improvement in post hoc assessments. Baseline scores varied across groups and were lowest in the CVPT group, which is likely why the CVPT group demonstrated the greater improvement over time. Additionally, the interventions provided to the CVPT group were most similar to the tasks utilized in the SOT. Specificity of training may have benefitted this group more greatly by helping train to the task. For example, the scenarios in VR training did not encompass an eyes closed condition. Future studies should employ and assess the effects of additional sensory feedback modalities, such as haptic feedback, to allow functional rehabilitative tasks in an eyes closed condition.

\subsection{Limitations}

There are several limitations in this study. First, final SOT assessments were not performed in 6 participants and resulted in data being carried forward to the final score. In the clinic where this trial was performed, participants that scored 70 points or better on the SOT were not required to repeat the assessment again. As a result, lack of additional data demonstrating further improvement may have biased the results toward the null hypothesis. Second, it is highly plausible that the sample size used in this pilot may not have been sufficient to demonstrate treatment main effects or treatment by time interactions, contributing to a type II error. However, confidence in the results is improved as the effect size estimates in calculations of Hedges's $g$ and $95 \%$ 
confidence intervals converged using the actual sample size and with the use of bootstrapping.

The participants and the clinicians who provided care in this study were not blinded to treatment allocation. A future study that employs a larger sample size, blinding of the assessing clinician, and allocation concealment is warranted. Lastly, the study sample was primarily composed of young adults with vestibular deficit following concussion. The generalizability of these findings is thus limited to individuals with a similar diagnosis and age.

\subsection{Clinical and practice implications}

The use of VR appears to be therapeutic in improving patient-reported, clinical, and instrumented measures of dizziness and function and may be complementary to standard care for individuals with central vestibular deficit following concussion. Due to the small sample size and potential sources of bias employed in this pilot study, clinical superiority cannot be established and will require further study for elucidation. When the potential clinical benefit of VR interventions is considered in the context of low anticipated risk, the use of VR in a comprehensive clinical treatment course should be considered. This point becomes especially salient when barriers to care preclude access to specialized conventional care. Furthermore, VR may have value when treatment tolerance and compliance to standard care modalities is poor.

In this study, non-specific VR scenarios were employed during treatment. As this line of study progresses, individualized scenarios designed to specifically target deficits identified during the history and physical examination is warranted. A multitude of iVREs can be used to create scenarios which challenge participants during physical therapy. In addition, the use of $360^{\circ}$ auditory stimulation within the VR scenarios could help elicit larger head or body turning that clinicians try to produce during therapy. The ability to customize treatment may help improve adherence and engagement to treatment, while optimizing self-efficacy and improving confidence during ecologically valid tasks. Though specific measures of self-efficacy were not included in the scope of this pilot study, future research should assess aspects of VR therapy in this population and how it affects not just perceived abilities, but also therapy compliance. Virtual reality exercises, which include activities in an eyes closed condition with and without haptic feedback, may also improve outcomes. In conclusion, CVPT, VRVPT, or a combination of both interventions results in improved patientreported, clinician-assessed, and instrumented measures of balance and function following six weeks of treatment. Incorporation of VR-based exercises in a comprehensive clinical treatment course could provide benefits for those with persistent vestibular impairments following concussion.

Acknowledgements Jose Dominguez, PT, PhD; Jacob VanDehy, MA; Jordan Sturdy, MA; Grant Meisenholder, DPT

Authors' contribution PS, KG, and AM contributed to the study conception and design. Material preparation, data collection, and analysis were performed by PS, JF, DB, KH, AM, JB, JJ and KG Statistical analyses were performed by JB, and JJ. All authors read and approved the final manuscript.

Funding The authors are military members and employees of the U.S. Government. This work was prepared as part of their official duties. Title 17, U.S.C. $\$ 105$ provides that copyright protection under this title is not available for any work of the U.S. Government. Title 17, U.S.C. $\$ 101$ defines a U.S. Government work as work prepared by a military service member or employee of the U.S. Government as part of that person's official duties. This work was supported by the U.S. Navy Bureau of Medicine and Surgery's Wounded, Ill, and Injured Program under work unit no. 60818 and N1703. The views expressed in this article are those of the authors and do not necessarily reflect the official policy or position of the Department of the Navy, Department of Defense, nor the U.S. Government. The study protocol was approved by the Naval Health Research Center Institutional Review Board in compliance with all applicable Federal regulations governing the protection of human subjects. Research data were derived from an approved Naval Health Research Center Institutional Review Board protocol number NHRC.2011.0027.

Data availability The data that support the findings of this study are available from the corresponding author, P.S., upon reasonable request.

\section{Declarations}

Conflicts of interest There are no conflicts of or competing interests.

Ethics approval The study protocol was approved by the Naval Health Research Center Institutional Review Board in compliance with all applicable Federal regulations governing the protection of human subjects. Research data were derived from an approved Naval Health Research Center Institutional Review Board protocol number NHRC.2011.0027.

Consent to participate All participants were informed of study procedures and gave their written consent to participate.

Consent for publication Use of data and materials for purposes of reporting and publishing findings was included in the informed consent procedures and all participants gave their written consent allowing use of their data for publication.

Open Access This article is licensed under a Creative Commons Attribution 4.0 International License, which permits use, sharing, adaptation, distribution and reproduction in any medium or format, as long as you give appropriate credit to the original author(s) and the source, provide a link to the Creative Commons licence, and indicate if changes were made. The images or other third party material in this article are included in the article's Creative Commons licence, unless indicated otherwise in a credit line to the material. If material is not included in the article's Creative Commons licence and your intended use is not permitted by statutory regulation or exceeds the permitted use, you will 
need to obtain permission directly from the copyright holder. To view a copy of this licence, visit http://creativecommons.org/licenses/by/4.0/.

\section{References}

Alahmari KA, Sparto PJ, Marchetti GF, Redfern MS, Furman JM, Whitney S (2014) Comparison of virtual reality based therapy with customized vestibular physical therapy for the treatment of vestibular disorders. IEEE Trans Neural Syst Rehabil Eng 22:389399. https://doi.org/10.1109/TNSRE.2013.2294904

Alsalaheen BA, Mucha A, Morris LO, Whitney SL, Furman JM, Camiolo-Reddy CE, Collins MW, Lovell MR, Sparto PJ (2010) Vestibular rehabilitation for dizziness and balance disorders after concussion. J Neurol Phys Ther 34:87-93. https://doi.org/10.1097/ NPT.0b013e3181dde568

Anderson T, Heitger M, Macleod AD (2006) Concussion and mild head injury. Pract Neurol 6:342-357. https://doi.org/10.1136/jnnp. 2006.106583

Badke MB, Miedaner JA, Grove CR, Shea TA, Pyle GM (2005) Effects of vestibular and balance rehabilitation on sensory organization and dizziness handicap. Ann Otol Rhinol Laryngol 114:48-54. https://doi.org/10.1177/000348940511400109

Basford JR, Chou LS, Kaufman KR, Brey RH, Walker A, Malec JF, Moessner AM, Brown AW (2003) An assessment of gait and balance deficits after traumatic brain injury. Arch Phys Med Rehabil 84:343-349. https://doi.org/10.1053/apmr.2003.50034

Bergeron M, Lortie CL, Guitton MJ (2015) Use of virtual reality tools for vestibular disorders rehabilitation: a comprehensive analysis. Adv Med. https://doi.org/10.1155/2015/916735

Cawthorne T (1944) The physiological basis for head exercises. J Chart Soc Physiother 3:106-107

Chong SF, Choo R (2011) Introduction to Bootstrap. Proceed Singapore Healthc. https://doi.org/10.1177/201010581102000314

Covassin T, Beidler E, Ostrowski J, Wallace J (2015) Psychosocial aspects of rehabilitation in sports. Clin Sports Med 34(2):199_ 212. https://doi.org/10.1016/j.csm.2014.12.004

Cuthbert J, Staniszewski K, Hays K, Gerber D, O'dell A, O'dell D. (2014) Virtual reality-based therapy for the treatment of balance deficits in patients receiving inpatient rehabilitation for traumatic brain injury. Brain Inj 28:181-188. https://doi.org/ 10.3109/02699052.2013.860475

Defense and Veterans Brain Injury Center (2019) DoD numbers for traumatic brain injury worldwide totals. https://dvbic.dcoe.mil/ sites/default/files/tbi-numbers/DVBIC_WorldwideTotal_20002019_Q3.pdf. Accessed 28 Apr 2020

Efron B (1987) Better bootstrap confidence intervals. J Am Stat Assoc 82(397):171-185. https://doi.org/10.1080/01621459. 1987.10478410

Gerlanc D, Kirby KN (2015) bootES: bootstrap effect sizes (Version 1.2). Computer software. https://cran.r-project.org/web/packa ges/bootES

Gottshall K, Drake A, Gray N, McDonald E, Hoffer ME (2003) Objective vestibular tests as outcome measures in head injury patients. Laryngoscope 113(10):1746-1750

Gottshall KR, Gray N, Drake A (2005) A unique collaboration of female medical providers within the United States armed forces: rehabilitation of a marine with post-concussive vestibulopathy. Work 24(4):381-386

Gottshall KR, Hoffer ME (2010) Tracking recovery of vestibular function in individuals with blast-induced head trauma using vestibular-visual-cognitive interaction tests. J Neurol Phys Ther 34(2):94-97
Gottshall KR (2011) Vestibular rehabilitation after mild traumatic brain injury with vestibular pathology. Neuro Rehabil 29:167171. https://doi.org/10.3233/NRE-2011-0691

Gottshall KR, Sessoms PH (2015) Improvements in dizziness and imbalance results from using a multi disciplinary and multi sensory approach to vestibular physical therapy-a case study. Front Syst Neurosci 9:106. https://doi.org/10.3389/fnsys.2015. 00106

Hall AM, Ferreira PH, Maher CG, Latimer J, Ferreira ML (2010) The influence of the therapist-patient relationship on treatment outcome in physical rehabilitation: a systematic review. Phys Ther 90(8):1099-1110. https://doi.org/10.2522/ptj.20090245

Haluch K, Bodell D, Sessoms P, Meisenholder G (2020) Conventional and virtual reality vestibular physical therapy exercise programs. figshare. J Contrib. https://doi.org/10.6084/m9.figsh are.12456683.v1

Hoffer ME, Gottshall KR, Moore R, Balough BJ, Wester D (2004) Characterizing and treating dizziness after mild head injury. Otol Neurotol 25:135-138

Hoffer ME, Gottshall K, Kopke RD, Weisskopf P, Moore R, Allen KA, Wester D (2003) Vestibular testing abnormalities in individuals with motion sickness. Otol Neurotol 24(4):633-636. https://doi.org/10.1097/00129492-200307000-00017

Horak FB, Jones-Rycewicz C, Black FO, Shumway-Cook A (1992) Effects of vestibular rehabilitation on dizziness and imbalance. Otolaryngol Head Neck Surg 106:175-180. https://doi.org/10. $1177 / 019459989210600220$

Howard MC (2017) A meta analysis and systemic literature review of virtual reality rehabilitation programs. Comput Hum Behav 70:317-327. https://doi.org/10.1016/j.chb.2017.01.013

Newman CW, Jacobson GP (1993) Application of self-report scales in balance function handicap assessment and management. Semin Hear 14(4):363-375

Johnson CM, Perez CF, Hoffer ME (2014) The implication of physical injury on otovestibular and cognitive symptomatology following blast exposure. Otolaryngol Head Neck Surg 150:437440. https://doi.org/10.1177/0194599813515184

Kassambara, A (2019) rstatix: Pipe-friendly framework for basic statistical tests

Lajoie Y, Gallagher SP (2004) Predicting falls within the elderly community: comparison of postural sway, reaction time, the Berg Balance Scale and the Activities-specific Balance Confidence Scale for comparing fallers and non-fallers. Arch Gerontol Geriatr 38:11-26. https://doi.org/10.1016/S0167-4943(03) 00082-7

Lee H, Sullivan J, Schneiders AG (2013) The use of the dual task paradigm in detecting gait performance deficits following a sports-related concussion: a systematic review and meta-analysis. J Sci Med Sports 16:2-7. https://doi.org/10.1016/j.jsams. 2012.03.013

Levin MF, Weiss PL, Keshner EA (2015) Emergence of virtual reality as a tool for upper limb rehabilitation incorporation of motor control and motor learning principles. Phys Ther 95:415-425. https://doi.org/10.2522/ptj.20130579

Pape MM, Kodosky PN, Hoover P (2019) The community balance and mobility scale: detecting impairment in military service members with mild traumatic brain injury. Mil Med 185(3-4):428-435

Pinheiro J, Bates D, DebRoy S, Sarkar D (2019) R Core Team. 2019. nlme: linear and nonlinear mixed effects models. R package version 3.1-141

Powell LE, Myers AM (1995) The activities-specific balance confidence (ABC) scale. J Gerontol A Biol Sci Med Sci 50(1):M28M34. https://doi.org/10.1093/gerona/50A.1.M28

Quatman-Yates CC, Hunter-Giordano A, Shimamura KK, Landel R, Alsalaheen BA, Hanke TA, et al (2020) Physical therapy 
evaluation and treatment after concussion/mild traumatic brain injury. J Orthop Sports Phys Ther 50:CPG1-CPG73. https://doi. org/10.2519/jospt.2020.0301

R Core Team (2012) R: A language and environment for statistical computing. R Foundation for Statistical Computing, Vienna, Austria. http://www.R-project.org/

Scherer MR, Schubert MC (2009) Traumatic brain injury and vestibular pathology as a comorbidity after blast exposure. Phys Ther 89:980-992. https://doi.org/10.2522/ptj.20080353

Shepard NT, Smith-Wheelock M, Telian SA, Raj A (1993) Vestibular and balance rehabilitation therapy. Ann Otol Rhinol Laryngol 102:198-205. https://doi.org/10.1177/000348949310200306

Teel EF, Register-Mihalik JK, Blackburn JT, Guskiewicz KM (2013) Balance and cognitive performance during a dual-task: preliminary implications for use in concussion assessment. J Sci Med Sport 16:190-194. https://doi.org/10.1016/j.jsams.2012.09.007

Torchiano M (2017) Effsize: efficient effect size computation. R Package Version 07:1

Whitney SL, Alghwiri A, Alghadir A (2015) Physical therapy for persons with vestibular disorders. Curr Opin Nerol 28:61-68. https://doi.org/10.1097/WCO.0000000000000162
Whitney SL, Sparto PJ, Hodges LF, Babu SV, Furman JM, Redfern MS (2006) Responses to a virtual grocery store in persons with and without vestibular dysfunction. CyberPsychol Behav 9:152-156. https://doi.org/10.1089/cpb.2006.9.152

Whitney SL, Wrisley DM, Brown KE, Furman JM (2004) Is perception of handicap related to functional performance in persons with vestibular dysfunction? Otol Neurotol 25(2):139-143

Wrisley DM, Marchetti GF, Kuharsky DK, Whitney SL (2004) Reliability, internal consistency, and validity of data obtained with the functional gait assessment. Phys Ther 84:906-918. https://doi.org/ 10.1093/ptj/84.10.906

Publisher's Note Springer Nature remains neutral with regard to jurisdictional claims in published maps and institutional affiliations. 